\title{
Non-Uniqueness in the Cauchy Problem for Partial Differential Operators with Multiple Characteristics, II
}

\author{
By \\ Shizuo NAKANE*
}

§ 0 . Introduction

In this paper, we shall consider non-uniqueness of $C^{\infty}$-solutions of the non-characteristic Cauchy problem for a class of operators with $C^{\infty}$-coefficients containing some degenerate elliptic operators. Then we shall extend the non-uniqueness results of our preceding papers [6] and [7].

A typical example of the operators treated here is the following operator in $\mathbb{R}^{2}$ :

$$
P=\left(\partial_{t}-\sqrt{-1} t^{l} C(t, x) \partial_{x}\right)^{p}+t^{k} A(t, x)\left(\sqrt{-1} \partial_{x}\right)^{q}-t^{m} B(t, x)\left(\sqrt{-1} \partial_{x}\right)^{q-r},
$$

where $p \geqq q>r \geqq 1$. Plis [10] treated the case $l=m=0, A=B=C=1$ (i.e. elliptic case) and Nakane [6] treated the case $A=B=C=1$ (i.e. degenerate elliptic case). These results show that under some conditions on $k, l, m$, there exist $C^{\infty}$-functions $u$ and $f$ satisfying

$$
P u-f u=0, \quad(0,0) \in \operatorname{supp} u \subset\{\mathrm{t} \geqq 0\} .
$$

An important property of $P$ is that the imaginary parts of its characteristic roots have finite order zeros on the initial surface $t=0$. For these operators, [6], Ōkaji [9], Roberts [11] and Uryu [13] showed uniqueness under Levi type conditions on the lower order terms. But they considered mainly the case of variable multiple characteristics. We are much interested in the case of constant multiplicity (i.e. $p>q)$. As for this case, there are few results. In [6] and [9], they treated the case $p=q=2, r=1$ and $A=0$. For first order operators, 
see Strauss-Treves [12] or Zuily [14].

The main purpose of this paper is to give a necessary condition for uniqueness on the lower order terms for operators of the above type. Then we conclude that uniqueness does not hold unless the lower order terms degenerate according to the order of degeneracy of the imaginary parts of characteristic roots. Another purpose of this paper is to investigate the effect of the behaviour of imaginary parts (or of real parts) of symbols of operators.

The method of proofs of our results is a modification of those of Alinhac-Zuily [1], Lascar-Zuily [5], Nakane [7] and Zuily [14]. That is, we shall construct the functions $u$ and $f$ by using the method of geometrical optics. Our results will show that this method is very powerful to get necessary conditions for uniqueness.

\section{§1. Statement of Results}

Let $P=P\left(t, x ; \partial_{t}, D_{x}\right)$ be the following operator of order $p$ in $\mathbb{R}^{d+1}$ :

$$
\begin{aligned}
P= & \left(\partial_{t}-t^{l} C\left(t, x ; D_{x}\right)\right)^{p}+t^{k} A\left(t, x ; D_{x}\right)-t^{m} B\left(t, x ; D_{X}\right) \\
& +\sum_{j=1}^{p} \sum_{i \leqq j} t^{m(j, i)} B_{j, i}\left(t, x ; D_{x}\right) \partial_{t}^{p-j} .
\end{aligned}
$$

Here $\partial_{t}=\partial / \partial t, \quad D_{x}=\frac{1}{\sqrt{-1}}\left(\partial / \partial x_{1}, \cdots, \partial / \partial x_{d}\right), k, l, m, m(j, i) \in \mathbb{N}=\{0,1,2$, $\cdots\}, A, B, C, B_{j, i}$ are partial differential operators, homogeneous order $q, q-r, 1, i$ with respect to $D_{x}$ respectively, whose coefficients are $C^{\infty}$ in $U$, an open neighborhood of the origin in $\mathbb{R}^{d+1}$ and $p \geqq q>r \geqq 1$. Let $\xi$ be the dual variable of $x$.

Then the following theorem is a corollary of Theorem 1.1 of [7].

Theorem 1.1. Suppose

$$
\begin{aligned}
& \frac{p r+q m}{q-r}<k<\frac{p r l+(p-q) m}{p-q+r}, \\
& m(j, i)>\frac{j k}{p}+\frac{(i p-j q)(k-m)}{p r} .
\end{aligned}
$$

We also assume that there exist $\xi^{0} \in \mathbb{R}^{d} \backslash\{0\}$ and a branch $D\left(\xi^{0}\right)$ of $\left\{B\left(0,0 ; \xi^{0}\right)-A\left(0,0 ; \xi^{0}\right)\right\}^{1 / p}$ satisfying

$$
\operatorname{Re} D\left(\xi^{0}\right)>0 \text {, }
$$




$$
\operatorname{Re}\left\{\left(\frac{A\left(0,0 ; \xi^{0}\right)}{B\left(0,0 ; \xi^{0}\right)-A\left(0,0 ; \xi^{0}\right)}+1-\frac{q}{r}\right) D\left(\xi^{0}\right)\right\}>0 .
$$

Then there exist an open neighborhood $U^{\prime}$ of the origin and $C^{\infty}$-functions $u$ and $f$ in $U^{\prime}$ such that

$$
P u-f u=0, \quad(0,0) \in \operatorname{supp} u \subset\{t \geqq 0\} .
$$

Remark 1.1. Assumption (1.2) is equivalent to assumption (1.9) or (1.13) of Theorem 2 of [6]. Hence this theorem is a generalization of Theorem 2 of [6].

Remark 1.2. As in Remark 1.4 of [7], we introduce Newton polygons. In the $(X, Y)$-plane, we plot the following points:

$$
\begin{aligned}
& R_{1}=(q / p,-1), \quad R_{2}=(0, k / p), \quad R_{3}=(r / p, m / p), \\
& R_{4}=(q / p-1, l), \quad P_{j, i}=(q / p-i / j, m(j, i) / j) .
\end{aligned}
$$

The first inequality of (1.2) implies that $R_{3}$ is located below the line passing through the points $R_{1}$ and $R_{2}$. The second inequality of (1.2) implies that $R_{4}$ is located above the line passing through the points $R_{2}$ and $R_{3}$. Assumption (1.3) implies that all the points $P_{j, i}$ are located above the line passing through the points $R_{2}$ and $R_{3}$. Hence above theorem is a corollary of Theorem 1.1 of [7].

Now we consider the case $k>\frac{p r l+(p-q) m}{p-q+r}$.

\section{Theorem 1.2. Suppose}

$$
\begin{aligned}
& k>\frac{p r l+(p-q) m}{p-q+r}, \\
& m<(l+1)(q-r)-p, \\
& m(j, i)>l j+\frac{m-p l}{p-q+r}(j-i) .
\end{aligned}
$$

We also assume that there exist $\xi^{0} \in \mathbb{R}^{d} \backslash\{0\}$ and a branch $B\left(0,0 ; \xi^{0}\right)^{1 / p}$ satisfying

$$
\begin{aligned}
& \operatorname{Re} C\left(0,0 ; \xi^{0}\right)+\operatorname{Re} B\left(0,0 ; \xi^{0}\right)^{1 / p}>0, \\
& p \operatorname{Re} C\left(0,0 ; \xi^{0}\right)+(q-r) \operatorname{Re} B\left(0,0 ; \xi^{0}\right)^{1 / p}<0 .
\end{aligned}
$$

Then the same conclusion as in Theorem 1.1 holds. 
Remark 1.3. In terms of Remark 1.2, assumption (1.7) implies that $R_{4}$ is located below the line passing through the points $R_{2}$ and $R_{3}$. Assumption (1.8) implies that $R_{3}$ is located below the line passing through the points $R_{1}$ and $R_{4}$. Assumption (1.9) implies that all the points $P_{j, i}$ are located above the line passing through the points $R_{3}$ and $R_{4}$.

Example. We consider the following operator in $\boldsymbol{R}^{2}$ :

$$
P=\left(\partial_{t}-t^{l} D_{x}\right)^{p}-t^{m} B(t, x) D_{x}^{q-r},
$$

where $B \in C^{\infty}(U)$ and $p \geqq q>r \geqq 1$. Since it corresponds to the case $A=B_{j, i}=0$, assumptions (1.7) and (1.9) are automatically satisfied. We assume (1.8) and we consider assumption (1.10) and (1.11). By considering the effect of the similarity transformation: $x \mapsto h x$ for some $h \in \boldsymbol{R}$, we have the following:

Case 1. When $p \geqq 3$, assumptions (1.10) and (1.11) are satisfied if $B(0,0) \neq 0$.

Case 2. When $p=q=2$ and $r=1$, assumptions (1.10) and (1.11) are satisfied if $B(0,0) \notin \boldsymbol{C} \backslash[0, \infty)$.

Remark 1.4. Consider the following operator in $\boldsymbol{R}^{2}$ :

$$
P=\left(\partial_{t}-t^{l} D_{x}\right)^{2}-t^{m} B(t, x) D_{x}+C(t, x),
$$

where $B, C \in C^{\infty}(U)$. In [6], we showed that uniqueness holds for $P$ if $m>l-1$. Recently Ōkaji [9] showed that uniqueness holds for $P$ if $m \geqq l-1$. Furthermore, Professor K. Watanabe pointed us that uniqueness holds for $P$ with $m<l-1$ if $B(t, x)>0$. Hence assumptions (1.8), (1.10) and (1.11) are indispensable.

Finally we consider the case $k=\frac{p r l+(p-q) m}{p-q+r}$.

Theorem 1.3. Suppose (1.8), (1.9) and

$$
k=\frac{p r l+(p-q) m}{p-q+r}
$$

We also assume that there exist $\xi^{0} \in \mathbb{R}^{d} \backslash\{0\}$ and a branch $D\left(\xi^{0}\right)$ of $\left\{B\left(0,0 ; \xi^{0}\right)-A\left(0,0 ; \xi^{0}\right)\right\}^{1 / p}$ satisfying 


$$
\operatorname{Re} C\left(0,0 ; \xi^{0}\right)+\operatorname{Re} D\left(\xi^{0}\right)>0,
$$

$$
\begin{aligned}
& p \operatorname{Re} C\left(0,0 ; \xi^{0}\right) \\
& \quad+\operatorname{Re}\left\{\left(\frac{(p-q+r)(m-k) A\left(0,0 ; \xi^{0}\right)}{B\left(0,0 ; \xi^{0}\right)-A\left(0,0 ; \xi^{0}\right)}+q-r\right) D\left(\xi^{0}\right)\right\}<0 .
\end{aligned}
$$

Then the same conclusion as in Theorem 1.1 holds.

Remark 1.5. Assumption (1.12) implies that $R_{4}$ is located on the line passing through the points $R_{2}$ and $R_{3}$.

Since the proof of Theorem 1.3 is similar to that of Theorem 1.2, we omit its proof.

\section{§2. Proof of Theorem 1.2}

Let $\omega$ be a sufficiently small open neighborhood of the origin in $\mathbb{R}^{d}$. We construct the function $u(t, x)$ as a superposition of functions $u_{n}(t, x)$, defined in $U_{n}=\left(b_{n+1}, b_{n-1}\right) \times \omega$, of the form:

$$
\begin{aligned}
u_{n}(t, x) & =\exp \left\{\sqrt{-1} \tau_{n}\left(\xi^{0} x-\frac{\sqrt{-1}}{l+1}\left(t^{l+1}-b_{n}^{l+1}\right)\right)\right\} \exp \left\{\phi\left(\frac{t}{b_{n}}, x, b_{n}\right)\right\} \\
& \times \exp \left\{-\gamma_{n}(x)\right\} w_{n}\left(\frac{t}{b_{n}}, x\right),
\end{aligned}
$$

so that the function $f=P u / u$ becomes $C^{\infty}$ near the origin. The above form of $u_{n}$ is a modification of the one in [7]. Considering the degenerate elliptic part $t^{l} C\left(t, x ; D_{x}\right)$ of $P$, we introduce a complex phase function $\xi^{0} x-\frac{\sqrt{-1}}{l+1}\left(t^{l+1}-b_{n}^{l+1}\right)$. Then a similar argument as in [7] works for this case.

This method was originally introduced by Cohen [3] and [10], etc. for a special type of operators. In order to treat more general class of operators, Hörmander [4] constructed $u_{n}$ by using the method of geometrical optics. In [1] and [14], they have developed his idea and have obtained a more systematic way to construct $u_{n}$. As in [7], we treat the case of higher multiplicity. Then we come across a new difficulty when we solve the transport equations.

Put $t=\delta s$, where $\delta$ is a small positive parameter. Then $P$ is 
transformed into

$$
\begin{aligned}
\tilde{P}= & \left(\delta^{-1} \partial_{s}-\delta^{l} s^{l} C\left(\delta s, x ; D_{x}\right)\right)^{p}+\delta^{k} s^{k} A\left(\delta s, x ; D_{x}\right) \\
& -\delta^{m} s^{m} B\left(\delta s, x ; D_{x}\right)+\sum_{j=1}^{p} \sum_{i \leq j} \delta^{m(j, i)+j-p} s^{m(j, i)} B_{j, i}\left(\delta s, x ; D_{x}\right) \partial_{s}^{p-j} .
\end{aligned}
$$

Let $T$ be a small open neighborhood of 1 in $\mathbb{R}$ and let $B^{\infty}$ be the set of functions $f=f(s, x, \delta)$ which are $G^{\infty}$ with respect to $(s, x)$ and satisfy

$$
\left|\partial_{s}^{j} D_{x}^{\alpha} f\right| \leqq C_{j, \alpha} \quad \text { in } T \times \omega
$$

for any $(j, \alpha) \in \mathbb{N}^{d+1}$ as $\delta$ tends to 0 . We construct the asymptotic solution $u=u(s, x, \delta)$ of the equation $\tilde{P} u=0$ in $T \times \omega$ in the form:

$$
\begin{aligned}
u(s, x, \delta) & =\exp \left\{\sqrt{-1} \tau\left(\xi^{0} x-\frac{\sqrt{-1}}{l+1}\left(t^{l+1}-\delta^{l+1}\right)\right)\right\} \exp \{\phi(s, x, \delta)\} \\
& \times \exp \{-\gamma(x, \delta)\} w(s, x, \delta),
\end{aligned}
$$

where

$$
\begin{aligned}
& \phi(s, x, \delta)=\sum_{j=1}^{N} \nu_{j} \phi_{j}(s, x, \delta), \quad \phi_{j} \in B^{\infty}, \\
& \gamma(x, \delta)=\sum_{j=1}^{N} \tilde{\gamma}_{j}(x, \delta)=\sum_{j=1}^{N} \nu_{j} \gamma_{j}(x, \delta), \quad \gamma_{j} \in B^{\infty}, \\
& \tau=\delta^{-d_{0}}, \\
& \nu_{j}=\delta^{-d_{j}}, \quad 1 \leqq j \leqq N, \\
& d_{0}>d_{1}>\cdots>d_{N}>0, \\
& N \in N,
\end{aligned}
$$

all these are determined later.

Let us consider

$$
I=\exp \left\{-\sqrt{-1} \tau\left(\xi^{0} x-\frac{\sqrt{-1}}{l+1}\left(t^{l+1}-\delta^{l+1}\right)\right)-\phi+\gamma\right\} \times \tilde{P} u .
$$

We determine $\phi$ and $\gamma$ so that $I$ shall be written in the form:

$$
I=\delta^{-p} \nu_{1}^{p-1}\left(L_{0} w+\delta^{\varepsilon} L_{1} w\right) \text {, }
$$

where

$$
\begin{aligned}
& L_{0}=C_{0}(s, x, \delta) \partial_{s}+C_{1}(s, x, \delta), \quad C_{0}, C_{1} \in B^{\infty}, \\
& C_{0}(s, x, \delta) \neq 0,
\end{aligned}
$$

and $L_{1}=L_{1}\left(s, x, \delta ; \partial_{s}, D_{x}\right)$ is a partial differential operator of order $p$ 
with coefficients in $B^{\infty}$ and $\varepsilon>0$ is an appropriate constant. We note that $\xi^{0} x-\frac{\sqrt{-1}}{l+1}\left(t^{l+1}-\delta^{l+1}\right)$ and $\phi$ are phase functions and that $\gamma$ is the normalization term.

We put

$$
\begin{aligned}
I_{1}= & \left\{\delta^{l} \tau s^{l}\left(1-C\left(\delta s, x ; \xi^{0}\right)+\delta^{-1} \nu_{1} \phi_{1, s}\right\}^{p}\right. \\
& +\delta^{k} \tau^{q} s^{k} A\left(\delta s, x ; \xi^{0}\right)-\delta^{m} \tau^{q-r} s^{m} B\left(\delta s, x ; \xi^{0}\right) \\
& +\sum_{j=1}^{p} \sum_{i \leqq j} \delta^{m(j, i)} \tau^{i} s^{m(j, i)} B_{j, i}\left(\delta s, x ; \xi^{0}\right)\left(\delta^{l} \tau s^{l}+\delta^{-1} \nu_{1} \phi_{1, s}\right)^{p-j}, \\
I_{g}= & \left\{\delta^{l} \tau s^{l}\left(1-C\left(\delta s, x ; \xi^{0}\right)\right)+\delta^{-1} \sum_{h=1}^{g} \nu_{h} \phi_{h, s}\right)^{p} \\
& +\delta^{k} s^{k} A\left(\delta s, x ; \tau \xi^{0}+\sqrt{-1} \sum_{h=1}^{g-1} \nu_{h}\left(\gamma_{h, x}-\phi_{h, x}\right)\right) \\
& -\delta^{m} s^{m} B\left(\delta s, x ; \tau \xi^{0}+\sqrt{-1} \sum_{h=1}^{g-1} \nu_{h}\left(\gamma_{h, x}-\phi_{h, x}\right)\right) \\
+ & \sum_{j=1}^{p} \sum_{i \leqq j}(\delta s)^{m(j, i)} B_{j, i}\left(\delta s, x ; \tau \xi^{0}+\sqrt{-1} \sum_{h=1}^{g-1} \nu_{h}\left(\gamma_{h, x}-\phi_{h, x}\right)\right) \\
& \quad \times\left(\delta^{l} \tau s^{l}+\delta^{-1} \sum_{h=1}^{g} \nu_{h} \phi_{h, s}\right)^{p-j}, \quad(2 \leqq g \leqq N) .
\end{aligned}
$$

First we determine $\phi_{1}$ so that $I_{1}=0$. Next we determine $\gamma_{1}$ from $\phi_{1}$. Then we find $\phi_{2}$ so that $I_{2}=0\left(\delta^{\varepsilon-p_{1}^{p-1}}\right)$ and find $\gamma_{2}$ from $\phi_{2}$. In the same way, once we have determined $\phi_{j}$ and $\gamma_{j}(1 \leqq i \leqq g)$, we find $\phi_{g+1}$ so that $I_{g+1}=O\left(\delta^{\varepsilon-p} \nu_{1}^{p-1}\right)$ and then find $\gamma_{g+1}$ from $\phi_{g+1}$. We shall see that there exists $N \in N$ such that $I_{N+1}=O\left(\delta^{\varepsilon-p_{\nu}^{p}-1}\right)$ even if we take $\phi_{N+1}=\gamma_{N+1}=0$. Then, by the choice of $\tau, \nu_{j}(1 \leqq j \leqq N)$, it is easy to see that $I$ is of the form (2.1).

\section{(2. a) Determination of $\phi_{1}$.}

Now we consider $I_{1}$. We set $\delta^{l} \tau=\delta^{-1} \nu_{1}$ and $\delta^{p l} \tau^{p}=\delta^{m} \tau^{q-r}$. That is, we have

$$
\begin{aligned}
\tau & =\delta^{\frac{m-p l}{p-q+r}}, & d_{0} & =\frac{p l-m}{p-q+r}, \\
\nu_{1} & =\tau \delta^{l+1}, & d_{1} & =d_{0}-(l+1) .
\end{aligned}
$$

From (1.8), we can easily see that $d_{0}>d_{1}>0$. Here we have, from (1.7) and (1.9),

$$
\delta^{k} \tau^{q}=O\left(\delta^{m} \tau^{q-r}\right)
$$




$$
\delta^{m(j, i)} \tau^{i}\left(\delta^{l} \tau\right)^{p-j}=O\left(\delta^{p l} \tau^{p}\right) .
$$

Then there exist a small constant $\varepsilon>0$ and $D_{1, j}(s, x, \delta) \in B^{\infty}(1 \leqq j \leqq p)$ such that

$$
\begin{gathered}
I_{1}=\delta^{-p} \nu_{1}^{p}\left[\left\{\phi_{1, s}+s^{l}\left(1-C\left(0, x ; \xi^{0}\right)\right)\right\}^{p}-s^{m} B\left(0, x ; \xi^{0}\right)\right. \\
\left.+\delta^{\varepsilon} \sum_{j=1}^{p} D_{1, j}(s, x, \delta) \phi_{1, s}^{p-j}\right] .
\end{gathered}
$$

Let $\phi_{1}$ be the solution of the Cauchy problem:

$$
\left\{\begin{array}{l}
\left\{\phi_{1, s}+s^{l}\left(1-C\left(0, x ; \xi^{0}\right)\right)\right\}^{p}-s^{m} B\left(0, x ; \xi^{0}\right)+\delta^{\varepsilon} \sum_{j=1}^{p} D_{1, j} \phi_{1, s}^{p-j}=0 \\
\left.\phi_{1}\right|_{s=1}=0
\end{array}\right.
$$

We note that

$$
\phi_{1, s}(1, x, 0)=-1+C\left(0, x ; \xi^{0}\right)+B\left(0, x ; \xi^{0}\right)^{1 / p},
$$

$$
\phi_{1, s s}(1, x, 0)=-l+l C\left(0, x ; \xi^{0}\right)+\frac{m}{p} B\left(0, x ; \xi^{0}\right)^{1 / p} \text {. }
$$

We put $\beta_{j, 1}(x, \delta)=\operatorname{Re} \phi_{j, s}(1, x, \delta)$ and $2 \beta_{j, 2}(x, \delta)=\operatorname{Re} \phi_{j, s s}(1, x, \delta)$, $(1 \leqq j \leqq N)$. Then, from (1.10), it follows that

$$
1+\beta_{1.1}(x, \delta)>0
$$

by taking $\delta$ and $\omega$ small if necessary. Furthermore, since $B\left(0,0 ; \xi^{0}\right)$, $C\left(0,0 ; \xi^{0}\right) \neq 0$ from (1.10) and (1.11), we have

$$
\phi_{1, s}+s^{l}\left(1-C\left(0, x, \xi^{0}\right)\right) \neq 0
$$

for $(s, x) \in T \times \omega$ and for small $\delta$.

\section{(2. b) Determination of $\gamma_{1}$.}

Put $\delta=b_{n}=n^{-\rho}, \tau=\tau_{n}=b_{n}^{-d_{0}}$ and $\nu_{1}=\nu_{1, n}=b_{n}^{-d_{1}}$, where $n \in N, n \geqq n_{0}, n_{0}$ is sufficiently large and $\rho>0$ is a constant determined later. For $t \in\left(b_{n+1}, b_{n-1}\right)$, we define

$$
\begin{aligned}
G_{1, n}(t, x) & =\frac{1}{l+1}\left\{\tau_{n}\left(t^{l+1}-b_{n}^{l+1}\right)-\tau_{n+1}\left(t^{l+1}-b_{n+1}^{l+1}\right)\right\} \\
& +\nu_{1, n} \operatorname{Re} \phi_{1}\left(\frac{t}{b_{n}}, x, b_{n}\right)-\nu_{1, n+1} \operatorname{Re} \phi_{1}\left(\frac{t}{b_{n+1}}, x, b_{n+1}\right) .
\end{aligned}
$$

Lemma 2.1. We put $m_{n}=\left(b_{n}+b_{n+1}\right) / 2, l_{n}=b_{n}-b_{n+1}$ and $I_{1 . n}(x)=$ $G_{1, n}\left(m_{n}, x\right)$. Then we have

$$
I_{1, n}(x) \sim-\left(1+\beta_{1,1}(x, 0)\right) \rho n^{\rho d_{1}-1} .
$$


Proof. Since $\left|\frac{t}{b_{n}}-1\right|,\left|\frac{t}{b_{n+1}}-1\right| \leqq$ const. $\cdot n^{-1}$ for $t \in\left(b_{n+1}, b_{n-1}\right)$ and for $n \geqq n_{0}$, we may take

$$
\operatorname{Re} \phi_{1}(s, x, \delta)=\beta_{1,1}(x, 0)(s-1)+\beta_{1,2}(x, 0)(s-1)^{2} .
$$

Then we have

$$
\begin{aligned}
I_{1, n}(x) & =\frac{1}{l+1}\left\{\tau_{n}\left(m_{n}-b_{n}\right) \sum_{j=0}^{l} m_{n}^{j} b_{n}^{l-j}-\tau_{n+1}\left(m_{n}-b_{n+1}\right) \sum_{j=0}^{l} m_{n}^{j} b_{n+1}^{l-j}\right\} \\
& +\beta_{1,1}(x, 0)\left\{\frac{\nu_{1, n}}{b_{n}}\left(m_{n}-b_{n}\right)-\frac{\nu_{1, n+1}}{b_{n+1}}\left(m_{n}-b_{n+1}\right)\right\} \\
& +\beta_{1,2}(x, 0)\left\{\frac{\nu_{1, n}}{b_{n}^{2}}\left(m_{n}-b_{n}\right)^{2}-\frac{\nu_{1, n+1}}{b_{n+1}^{2}}\left(m_{n}-b_{n+1}\right)^{2}\right\} \\
& =\text { (1) + (2) + (3). }
\end{aligned}
$$

Since

$$
\begin{aligned}
\text { (1) } & \sim-\rho n^{\rho d_{1}-1}, \\
\text { (2) } & =-\frac{1}{2} \beta_{1,1}(x, 0) l_{n}\left(\frac{\nu_{1, n}}{b_{n}}+\frac{\nu_{1, n+1}}{b_{n+1}}\right)-\beta_{1,1}(x, 0) \rho n^{\rho d_{1}-1}, \\
|(3)| & \leqq \frac{1}{4}\left|\beta_{1,2}(x, 0)\right| l_{n}^{2}\left(\frac{\nu_{1, n+1}}{b_{n+1}^{2}}-\frac{\nu_{1, n}}{b_{n}^{2}}\right) \leqq \text { const. } \cdot n^{\rho d_{1}-3},
\end{aligned}
$$

we have (2.9). This completes the proof.

We define $\gamma_{1, n}(x)$ by

$$
\gamma_{1, n}(x)=-\sum_{j=n_{0}}^{n-1} I_{1, j}(x) \sim d_{1}^{-1}\left(1+\beta_{1,1}(x, 0)\right) n^{\rho d_{1}} .
$$

It is easy to see that there exists $\gamma_{1}(x, \delta) \in B^{\infty}$ satisfying

$$
\gamma_{1, n}(x)=\nu_{1, n} \gamma_{1}\left(x, b_{n}\right)=\tilde{\gamma}_{1}\left(x, b_{n}\right) \text {. }
$$

Note that, from the definition,

$$
\gamma_{1, n+1}(x)-\gamma_{1, n}(x)=-I_{1, n}(x) \text {. }
$$

(2. c) Determination of $\phi_{j}, \gamma_{j}(j \geqq 2)$.

In order to determine $\phi_{2}$, we consider $I_{2}$. Since $I_{1}=0$, we have

$$
\begin{aligned}
I_{2}=\delta^{-p} \sum_{h=1}^{p}\left(\begin{array}{l}
p \\
h
\end{array}\right) \nu_{1}^{p-h} \nu_{2}^{h}\left\{s^{l}(1-C)+\phi_{1, s}\right\}^{p-h} \phi_{2, s}^{h} \\
+\sum_{\beta \neq 0} \frac{1}{\beta !}\left\{\delta^{k} s^{k} A^{(\beta)}\left(\delta s, x ; \tau \xi^{0}\right)-\delta^{m} s^{m} B^{(\beta)}\left(\delta s, x ; \tau \xi^{0}\right)\right\} \\
\quad \times\left\{\sqrt{-1} \nu_{1}\left(\gamma_{1, x}-\phi_{1, x}\right)\right\}^{\beta}
\end{aligned}
$$




$$
\begin{aligned}
+ & \sum_{j=1}^{p} \sum_{i \leqq j}(\delta s)^{m(j, i)} \sum_{\beta \neq 0} \frac{1}{\beta !} B_{j, i}^{(\beta)}\left(\delta s, x ; \tau \xi^{0}\right) \\
& \times\left\{\sqrt{-1} \nu_{1}\left(\delta_{1, x}-\phi_{1, x}\right)\right\}^{\beta}\left(\tau \delta^{l} s^{l}+\delta^{-1} \sum_{h=1}^{2} \nu_{h} \Lambda_{h, s}\right)^{p-j} \\
+ & \sum_{j=1}^{p} \sum_{i \leqq j}(\delta s)^{m(j, i)} B_{j, i}\left(\delta s, x ; \xi^{0}\right) \\
& \times \sum_{h=1}^{p-j}(p-j)\left(\delta^{-1} \nu_{1}\right)^{p-j-h}\left(\delta^{-1} \nu_{2}\right)^{h}\left(s^{l}+\phi_{1, s}\right)^{p-j-h} \phi_{2, s}^{h} \\
= & (1)+\text { (2) + (3) + (4), }
\end{aligned}
$$

where

$$
\begin{aligned}
& C=C\left(0, x, \xi^{0}\right), \\
& A^{(\beta)}(t, x ; \xi)=\partial_{\xi}^{\beta} A(t, x ; \xi), \\
& \left\{\sqrt{-1} \nu_{1}\left(\gamma_{1, x}-\phi_{1, x}\right)\right\}^{\beta}=\prod_{h=1}^{d}\left\{\sqrt{-1} \nu_{1}\left(\gamma_{1, x_{h}}-\phi_{1, x_{h}}\right)\right\}^{\beta_{h}}
\end{aligned}
$$

and so on.

Here, by a direct calculation, we have

$$
\begin{aligned}
& \text { (1) }=\delta^{-p} \nu_{1}^{p-1} \nu_{2} \sum_{h=1}^{p}\left(\begin{array}{l}
p \\
h
\end{array}\right)\left(\nu_{2} / \nu_{1}\right)^{h-1}\left\{\phi_{1, s}+s^{l}(1-G)\right\}^{p-h} \phi_{2, s}^{h}, \\
& \text { (2) }=\delta^{m} \tau^{q-r-1} \nu_{1} D_{2}(s, x, \delta), \\
& \text { (3) }=\delta^{\varepsilon-p} \nu_{1}^{p+1} \tau^{-1} \sum_{h=0}^{p-1}\left(\nu_{2} / \nu_{1}\right)^{h} D_{2, h}(s, x, \delta) \phi_{2, s}^{h}, \\
& \text { (4) }=\delta^{\varepsilon-p} \nu_{1}^{p} \sum_{h=1}^{p-1}\left(\nu_{2} / \nu_{1}\right)^{h} D_{2, h}^{\prime}(s, x, \delta) \phi_{2, s}^{h},
\end{aligned}
$$

where $D_{2}, D_{2, h}, D_{2, h}^{\prime} \in B^{\infty}$.

If $\delta^{m} \tau^{q-r-1} \nu_{1}=0\left(\delta^{-p_{1}^{p-1}}\right)$, we take $\phi_{j}=0$ for $j \geqq 2$. Then the remainder term (2) is absorbed in $C_{1}$ of (2.2). If not so, we set $\delta^{-p_{\nu}}{ }_{1}^{p-1} \nu_{2}=\delta^{m} \tau^{q-r-1} \nu_{2}$, which implies $\nu_{2}=\tau^{-1} \nu_{1}^{2}=\delta^{d_{0}-2 d_{1}}$ and $d_{2}=2 d_{1}-d_{0}=d_{1}+\left(d_{1}-d_{0}\right)<d_{1}$. Then we have

$$
\begin{aligned}
I_{2} & =\delta^{-p} \nu_{1}^{p-1} \nu_{2}\left[p\left\{\phi_{1, s}+s^{l}(1-G)\right\}^{p-1} \phi_{2, s}\right. \\
& +\sum_{h=2}^{p}\left(\begin{array}{l}
p \\
h
\end{array}\right) \delta^{\left(d_{0}-d_{1}\right)(h-1)}\left\{\phi_{1, s}+s^{l}(1-C)\right\}^{p-h} \phi_{2, s}^{h} \\
& +D_{2}(s, x, \delta) \\
& +\delta^{\varepsilon} \sum_{h=0}^{p-1} \delta^{\left(d_{0}-d_{1}\right) h} D_{2, h}(s, x, \delta) \phi_{2, s}^{h}
\end{aligned}
$$




$$
\left.+\delta^{\varepsilon} \sum_{h=1}^{p-1} \delta^{\left(d_{0}-d_{1}\right)(h-1)} D_{2, h}^{\prime}(s, x, \delta) \phi_{2, s}^{h}\right]
$$

We construct $\phi_{2}$ so that $I_{2}=O\left(\delta^{\varepsilon-p_{1}^{p-1}}\right)$ and $\left.\phi_{2}\right|_{s=1}=0$. Such $\phi_{2}$ really exists. In fact, if we take $\varepsilon>0$ sufficiently small and take $\phi_{2}$ in the form:

$$
\left\{\begin{array}{l}
\phi_{2}(s, x, \delta)=\sum_{j=0}^{N_{2}} \delta^{\varepsilon j} \phi_{2, j}(s, x, \delta), \quad \text { for some } N_{2} \in N, \\
\left.\phi_{2}\right|_{s=1}=0,
\end{array}\right.
$$

then $\phi_{2, j}$ are determined successively. Here we use (2.9).

Now we construct $\gamma_{2}$ from $\phi_{2}$ in the same way as we have constructed $\gamma_{1}$ from $\phi_{1}$. We define for $t \in\left(b_{n+1}, b_{n-1}\right)$

$$
\begin{aligned}
& G_{2, n}(t, x)=\nu_{2, n} \operatorname{Re} \phi_{2}\left(\frac{t}{b_{n}}, x, b_{n}\right)-\nu_{2, n+1} \operatorname{Re} \phi_{2}\left(\frac{t}{b_{n+1}}, x, b_{n+1}\right), \\
& I_{2, n}(x)=G_{2, n}\left(m_{n}, x\right) .
\end{aligned}
$$

Then we have

Lemma 2.2. $I_{2, n}(x) \sim-\beta_{2,1}(x, 0) \rho n^{\rho d_{2}-1}$.

Since this lemma can be proved in the same way as Lemma 2.1, we omit the proof.

We define $\gamma_{2, n}(x)$ by

$$
\gamma_{2, n}(x)=-\sum_{j=n_{0}}^{n-1} I_{2, n}(x) \sim d_{2}^{-1} \beta_{2,1}(x, 0) n^{\rho d}
$$

Then it is easy to see that there exists $\gamma_{2}(x, \delta) \in B^{\infty}$ satisfying

$$
\gamma_{2, n}(x)=\nu_{2, n} \gamma_{2}\left(x, b_{n}\right)=\tilde{\gamma}_{2}\left(x, b_{n}\right) \text {. }
$$

Suppose we have constructed $\phi_{j}$ and $\gamma_{j}(1 \leqq j \leqq g)$. Then $I_{g}=$ $O\left(\delta^{\varepsilon-p} \nu_{1}^{p-1}\right)$ and we have

$$
\begin{aligned}
I_{g+1} & =\delta^{-p} \sum_{j=1}^{p}\left(\begin{array}{l}
p \\
j
\end{array}\right)\left\{\nu_{1} s^{l}(1-C)+\sum_{h=1}^{g} \nu_{h} \phi_{h, s}\right\}^{p-j_{\nu} \nu_{g+1}^{j} \phi_{g+1_{o} s}^{j}} \\
& +\sum_{\beta \neq 0} \frac{1}{\beta !}\left\{\delta^{k} s^{k} A^{(\beta)}\left(\delta s, x ; \tau \xi^{0}+\sqrt{-1} \sum_{h=1}^{g-1} \nu_{h}\left(\gamma_{h, x}-\phi_{h, x}\right)\right)\right.
\end{aligned}
$$




$$
\begin{gathered}
\left.-\delta^{m} s^{m} B^{(\beta)}\left(\delta s, x ; \tau \xi^{0}+\sqrt{-1} \sum_{h=1}^{g-1} \nu_{h}\left(\gamma_{h, x}-\phi_{h, x}\right)\right)\right\} \\
\times\left\{\sqrt{-1} \nu_{g}\left(\gamma_{g, X}-\phi_{g, X}\right)\right\}^{\beta} \\
+\sum_{j=1}^{p} \sum_{i \leqq j}(\delta s)^{m(j, i)} \sum_{\beta \neq 0} \frac{1}{\beta !} B_{j, i}^{(\beta)}\left(\delta s, x ; \tau \xi^{0}+\sqrt{-1} \sum_{h=1}^{g-1} \nu_{h}\left(\gamma_{h, x}-\phi_{h, x}\right)\right) \\
\quad \times\left\{\sqrt{-1} \nu_{g}\left(\gamma_{g, x}-\phi_{g, x}\right)\right\}^{\beta} \delta^{j-p}\left(\nu_{1} s^{l}+\sum_{h=1}^{g+1} \nu_{h} \phi_{h, s}\right)^{p-j} \\
+\sum_{j=1}^{p} \sum_{i \leqq j}(\delta s)^{m(j, i)} \delta^{j-p} B_{j, i}\left(\delta s, x ; \tau \xi^{0}+\sqrt{-1} \sum_{h=1}^{g-1} \nu_{h}\left(\gamma_{h, x}-\phi_{h, x}\right)\right) \\
\quad \times \sum_{h=1}^{p-j}(p-j)\left(\nu_{1} s^{l}+\sum_{h=1}^{g-1} \nu_{h} \phi_{h, s}\right)^{p-j-h} \nu_{g+1}^{h} \phi_{g+1, s}^{h} \\
+O\left(\delta^{\varepsilon-p} \nu_{1}^{p-1}\right) \\
=\text { (1) }+ \text { (2) }+ \text { (3)+ (4) }+ \text { (5). }
\end{gathered}
$$

By a direct calculation, we have

$$
\begin{aligned}
& \text { (1) }=\delta^{-p} \nu_{1}^{p-1} \nu_{g+1} \sum_{j=1}^{p}\left(\begin{array}{l}
p \\
j
\end{array}\right)\left\{s^{l}(1-C)+\sum_{h=1}^{g} \frac{\nu_{h}}{\nu_{1}} \phi_{h, s}\right\}^{p-j}\left(\frac{\nu_{g+1}}{\nu_{1}}\right)^{j-1} \phi_{g+1, s}^{j}, \\
& \text { (2) }=\delta^{m} \tau^{q-r-1} \nu_{g} D_{g+1}(s, x, \delta), \\
& \text { (3) }=\delta^{\varepsilon-p} \tau^{-1} \nu_{1}^{p} \nu_{g} \sum_{h=0}^{p-1}\left(\frac{\nu_{g+1}}{\nu_{1}}\right)^{h} D_{g+1, h}(s, x, \delta) \phi_{g+1, s}^{h}, \\
& \text { (4) }=\delta^{\varepsilon-p} \nu_{1}^{p} \sum_{h=1}^{p-1}\left(\frac{\nu_{g+1}}{\nu_{1}}\right)^{h} D_{g+1, h}^{\prime}(s, x, \delta) \phi_{g+1, s}^{h},
\end{aligned}
$$

where $D_{g+1}, D_{g+1, h}, D_{g+1, h}^{\prime} \in B^{\infty}$.

If $\delta^{m} \tau^{q-r-1} \nu_{g}=O\left(\delta^{-p} \nu_{1}^{p-1}\right)$, we take $\phi_{j}=\gamma_{j}=0$ for $j \geqq g+1$. Then the remainder term (2) is absorbed in $C_{1}$ of (2.2). If not so, we set $\delta^{-p} \nu_{1}^{p-1} \nu_{g+1}=\delta^{m} \tau^{q-r-1} \nu_{g}$, which means $\nu_{g+1}=\tau^{-1} \nu_{1} \nu_{g}$ and $d_{g+1}=d_{g}-\left(d_{0}-d_{1}\right)$ $<d_{g}$. Consequently we have

$$
\begin{aligned}
I_{g+1} & =\delta^{-p} \nu_{1}^{p-1} \nu_{g+1}\left[p\left\{s^{l}(1-C)+\sum_{h=1}^{g} \frac{\nu_{h}}{\nu_{1}} \phi_{h, s}\right\}^{p-1} \phi_{g+1, s}\right. \\
& +\sum_{j=2}^{p}\left(\begin{array}{c}
p \\
j
\end{array}\right)\left\{s^{l}(1-G)+\sum_{h=1}^{g} \frac{\nu_{h}}{\nu_{1}} \phi_{h, s}\right\}^{p-j}\left(\frac{\nu_{g+1}}{\nu_{1}}\right)^{j-1} \phi_{g+1, s}^{j} \\
& +D_{g+1}(s, x, \delta) \\
& +\delta^{\varepsilon} \sum_{h=0}^{p-1}\left(\frac{\nu_{g+1}}{\nu_{1}}\right)^{h} D_{g+1, h}(s, x, \delta) \phi_{g+1, s}^{h} \\
& \left.+\delta^{\varepsilon} \sum_{h=1}^{p-1}\left(\frac{\nu_{g+1}}{\nu_{1}}\right)^{h-1} D_{g+1, h}(s, x, \delta) \phi_{g+1}^{h}\right] \\
& +O\left(\delta^{\varepsilon-p_{\nu}^{p-1}}\right) .
\end{aligned}
$$

We take $\phi_{g+1}$ so that $I_{g+1}=O\left(\delta^{\varepsilon-p} \nu_{1}^{p-1}\right)$ and $\left.\phi_{g+1}\right|_{s=1}=0$. By the same 
way as before, we can construct such $\phi_{g+1}$. We can also construct $\gamma_{g+1}$ from $\phi_{g+1}$ as before.

Since $d_{g}=d_{g-1}-\left(d_{0}-d_{1}\right)=\cdots=d_{0}-g\left(d_{0}-d_{1}\right)=d_{0}-g(l+1)$, there exists $N \in N$ such that $I_{N+1}=O\left(\delta^{\varepsilon-p} \nu_{1}^{p-1}\right)$ even if we take $\phi_{N+1}=\gamma_{N+1}=0$.

It is easy to see that the remainder terms of $I$ are absorbed in the term $\delta^{\varepsilon} L_{1} w$ of (2.1) by taking $\varepsilon$ small if necessary. We remark that, from (2.8),

$$
C_{0}(s, x, \delta)=p\left\{s^{l}(1-C)+\sum_{h=1}^{N} \frac{\nu_{h}}{\nu_{1}} \phi_{h, s}\right\}^{p-1} \neq 0
$$

for sufficiently small $\delta$, which implies (2.3).

(2. d) Transport equations.

Now we consider the formal solution of the equation $L_{0} w+\delta^{\varepsilon} L_{1} w=0$. Let $w_{j}(j \geqq 0)$ be the solutions of the following equations :

$$
\left\{\begin{array} { l } 
{ L _ { 0 } w _ { 0 } = 0 , } \\
{ w _ { 0 } | _ { s = 1 } = 1 , }
\end{array} \quad \left\{\begin{array}{l}
L_{0} w_{j}=-L_{1} w_{j-1}, \\
\left.w_{j}\right|_{s=1}=0 .
\end{array} \quad(j \geqq 1)\right.\right.
$$

It is easy to see that there exists a function $g=g(s, x, \delta, \eta)$ such that for any $(j, \alpha) \in \mathbb{N}^{d+1}, K \in \mathbb{N}$, there exists $C=C_{j, \alpha, K}>0$ satisfying

$$
\left|\partial_{s}^{j} D_{x}^{\alpha}\left(g-\sum_{i=0}^{K-1} \eta^{i} w_{i}\right)\right| \leqq C|\eta|^{K}
$$

Then $w=w(s, x, \delta)=g\left(s, x, \delta, \delta^{\varepsilon}\right)$ is the desired solution.

We set $w_{n}(s, x)=w\left(s, x, b_{n}\right)$ and $\gamma_{n}(x)=\gamma\left(x, b_{n}\right)$ and we define in $U_{n}$,

$$
\begin{aligned}
v_{n}(t, x)= & \exp \left\{\sqrt{-1} \tau_{n}\left(\xi^{0} x-\frac{\sqrt{-1}}{l+1}\left(t^{l+1}-b_{n}^{l+1}\right)\right)\right\} \exp \left\{\phi\left(\frac{t}{b_{n}}, x, b_{n}\right)\right\} \\
& \times \exp \left\{-\gamma_{n}(x)\right\} w_{n}\left(\frac{t}{b_{n}}, x\right) .
\end{aligned}
$$

By the argument above, we have

Proposition 2.3. In $U_{n}$, we define $f_{n}=P v_{n} / v_{n}$. Then, for any $(j, \alpha)$ $\in N^{d+1}$, there exists a>0 such that for any $K \in N$ there exists $C=C_{j, \alpha, K}$ $>0$ satisfying

$$
\left|\partial_{t}^{j} D_{x}^{\alpha} f_{n}\right| \leqq C n^{-a K}
$$

for $(t, x) \in U_{n}$ and for $n \geqq n_{0}$. 
(2. e) The set where $\left|v_{n}\right|=\left|v_{n+1}\right|$.

In $U_{n}$, we put $F_{n}(t, x)=\log \left|\frac{v_{n}}{v_{n+1}}\right|$. Then we have

Proposition 2. 4. Suppose $\rho>2 / d_{1}$. Then there exist $G>0$ and $\sigma>\rho+1$ such that

$$
\frac{\partial F_{n}}{\partial t} \geqq C n^{\sigma}
$$

for $(t, x) \in U_{n}$ and for $n \geqq n_{0}$.

Proof. As before, we may take

$$
\operatorname{Re} \phi(s, x, \delta)=\sum_{j=1}^{N} \nu_{j}\left\{\beta_{j, 1}(x, 0)(s-1)+\beta_{j, 2}(x, 0)(s-1)^{2}\right\} .
$$

Then it follows that

$$
\begin{aligned}
\frac{\partial F_{n}}{\partial t}= & t^{l}\left(\tau_{n}-\tau_{n+1}\right)+\sum_{j=1}^{N} \beta_{j, 1}(x, 0)\left(\frac{\nu_{j, n}}{b_{n}}-\frac{\nu_{j, n+1}}{b_{n+1}}\right) \\
& +\sum_{j=1}^{N} 2 \beta_{j, 2}(x, 0)\left\{\frac{\nu_{j, n}}{b_{n}^{2}}\left(t-b_{n}\right)-\frac{\nu_{j, n+1}}{b_{n+1}^{2}}\left(t-b_{n+1}\right)\right\} \\
& +\left(\frac{w_{n, s}}{w_{n}} b_{n}^{-1}-\frac{w_{n+1, s}}{w_{n+1}} b_{n+1}^{-1}\right) \\
= & \text { (1) }+ \text { (2) + (3) }+ \text { (4). }
\end{aligned}
$$

Here

$$
\begin{aligned}
& \text { (1) - } \rho d_{0} n^{\rho\left(d_{1}+1\right)-1} \text {, } \\
& \text { (2) } \sim-\sum_{j=1}^{N} \beta_{j, 1}(x, 0) \rho\left(d_{j}+1\right) n^{\left(\rho d_{j}+1\right)-1} \\
& \sim-\beta_{1,1}(x, 0) \rho\left(d_{1}+1\right) n^{\rho\left(d_{1}+1\right)-1}, \\
& \text { (3) }=-\sum_{j=1}^{N} 2 \beta_{j, 2}(x, 0)\left\{\frac{\nu_{j, n+1}}{b_{n+1}^{2}}\left(b_{n}-b_{n+1}\right)+\left(t-b_{n}\right)\left(\frac{\nu_{j, n+1}}{b_{n+1}^{2}}-\frac{\nu_{j, n}}{b_{n}^{2}}\right)\right. \\
& \sim-\sum_{j=1}^{N} 2 \beta_{j, 2}(x, 0)\left\{\rho n^{\rho\left(d_{j}+1\right)-1}+O\left(n^{\rho\left(d_{j}+1\right)-2}\right)\right\} \\
& \sim-2 \beta_{1,2}(x, 0) n^{\rho\left(d_{1}+1\right)-1} \\
& \text { (4) } \mid \leqq \text { const. } \cdot n^{\rho} \text {. }
\end{aligned}
$$

If we take $\rho>2 / d_{1}$, we have $\rho\left(d_{1}+1\right)-1>\rho+1$. Hence, if we put $\sigma=\rho\left(d_{1}+1\right)-1>\rho+1$, we have

$$
\frac{\partial F_{n}}{\partial t} \sim-\rho\left\{d_{0}+\beta_{1,1}(x, 0)\left(d_{1}+1\right)+2 \beta_{1,2}(x, 0)\right\} n^{\sigma} .
$$


From assumption (1.11) and (2.5), (2.6), it follows that

$$
\begin{aligned}
& -\left\{d_{0}+\beta_{1,1}(0,0)\left(d_{1}+1\right)+2 \beta_{1,2}(0,0)\right\} \\
& =-\frac{p l-m}{p-q+r}\left\{p \operatorname{Re} C\left(0,0 ; \xi^{0}\right)+(q-r) \operatorname{Re} B\left(0,0 ; \xi^{0}\right)\right\} \\
& >0 .
\end{aligned}
$$

Hence, if we take $\omega$ sufficiently small, there exists $G>0$ such that

$$
\frac{\partial F_{n}}{\partial t} \geqq C n^{\alpha} \quad \text { in } U_{n} \text { 。 }
$$

This completes the proof.

By virtue of (2.13) and the implicit function theorem, there exists $m_{n}(x) \in C^{\infty}(\omega)$ satisfying

$$
F_{n}\left(m_{n}(x), x\right)=0 .
$$

Lemma 2.5. For sufficiently large $n, m_{n}(x) \in\left(b_{n+1}, b_{n-1}\right)$.

Proof. There exists $m_{n}^{*}(x)$ for each $x \in \omega$, satisfying

$$
m_{n}(x)-m_{n}=\frac{F_{n}\left(m_{n}(x), x\right)-F_{n}\left(m_{n}, x\right)}{\frac{\partial F_{n}}{\partial t}\left(m_{n}^{*}(x), x\right)} .
$$

From the choice of $\gamma_{n}$, it follows that $F_{n}\left(m_{n}, x\right)=O(1)$. Hence, from (2.13) and (2.14), we have

$$
\left|m_{n}(x)-m_{n}\right| \leqq \text { const. } \cdot n^{-\sigma} \text {. }
$$

Since $\sigma>\rho+1, m_{n}(x) \in\left(b_{n+1}, b_{n-1}\right)$ for sufficiently large $n$. This completes the proof.

(2. f) Modification of $v_{n}$.

We set

$$
\begin{aligned}
u_{n}(t, x)= & \exp \left\{\sqrt{-1} \tau_{n}\left(\xi^{0} x-\frac{\sqrt{-1}}{l+1}\left(t^{l+1}-b_{n}^{l+1}\right)\right)\right\} \exp \left\{\phi\left(\frac{t}{b_{n}}, x, b_{n}\right)\right\} \\
& \times \exp \left\{-\gamma_{n}(x)\right\}\left\{w_{n}\left(\frac{t}{b_{n}}, x\right)+z_{n}\left(\frac{t}{b_{n}}, x\right)\right\} .
\end{aligned}
$$

As in the proof of Proposition 3.5 of [7] or in the proof of fundamental lemma of [1], we have the following proposition. 
Proposition 2.6. Put $S_{n}=\left\{t=m_{n}(x)\right\}$. Then there exists $z_{n} \in B^{\infty}$ such that
(i) $\quad \tilde{F}_{n}=\log \left|\frac{u_{n}}{u_{n+1}}\right|$ satisfies (2.13) and (2.14),
(ii) $g_{n}=P u_{n} / u_{n}$ satisfies (2.12),
(iii) $g_{n}$ is flat on $S_{n}$ and on $S_{n-1}$.

Remark 2.1. In order to construct $z_{n}$, we use Whitney's extension theorem with estimates, which can be easily proved (see [1] or [7]).

(2.g) Smoothness of $u$ and $f$.

Let $\chi \in C_{0}^{\infty}(\boldsymbol{R})$ be a function satisfying

$$
\chi(s)=1 \quad \text { for }|s| \leqq 3 / 4, \quad \operatorname{supp} \chi \subset[-1,1] \text {, }
$$

and put $\chi_{n}(t)=\chi\left(\frac{t-b_{n}}{l_{n}}\right)$.

We define the desired functions $u$ and $f$ by

$$
\begin{aligned}
& u(t, x)= \begin{cases}\sum_{n \geqq n_{0}} \chi_{n}(t) u_{n}(t, x) & t>0, \\
0 & t \leqq 0,\end{cases} \\
& f(t, x)= \begin{cases}P u / u & t>0, \\
0 & t \leqq 0 .\end{cases}
\end{aligned}
$$

First we show the smoothness of $f$. Note that, from the above definition, $f$ may fail to be smooth only on $t=0$ or on $S_{n}$.

(2. g. 1) For $t \in\left[b_{n}-\frac{3}{4} l_{n}, b_{n+1}+\frac{3}{4} l_{n+1}\right]$, we have

$$
f=\frac{P\left(u_{n}+u_{n+1}\right)}{u_{n}+u_{n+1}}=\frac{g_{n} u_{n}+g_{n+1} u_{n+1}}{u_{n}+u_{n+1}} .
$$

From (2.15), we can easily see that $m_{n}(x) \in\left[b_{n}-\frac{3}{4} l_{n}, b_{n+1}+\frac{3}{4} l_{n+1}\right]$. If $t \geqq m_{n}(x),\left|u_{n}\right| \geqq\left|u_{n+1}\right|$ and

$$
\left|u_{n}+u_{n+1}\right| \geqq\left|u_{n}\right|\left(1-\left|\frac{u_{n+1}}{u_{n}}\right|\right)=\left|u_{n}\right|\left(1-e^{-\tilde{F}_{n}}\right) .
$$

Using the inequality: 


$$
1-e^{-x} \geqq \begin{cases}x / 4 & \text { for } x \in[0,2] \\ 1 / 2 & \text { for } x \geqq 1\end{cases}
$$

and (2.13), we have

$$
\left|u_{n}+u_{n+1}\right| \geqq\left\{\begin{array}{l}
C n^{\sigma}\left(t-m_{n}(x)\right)\left|u_{n}\right| \\
\left|u_{n}\right| / 2 \quad \text { if } \tilde{F}_{n} \geqq 1 .
\end{array} \text { if } \tilde{F}_{n} \in[0,2],\right.
$$

Since $g_{n}$ and $g_{n+1}$ are flat on $t=m_{n}(x)$, this implies that $f$ is $C^{\infty}$ on $S_{n}$ and that

$$
|f| \leqq \max \left\{\frac{\left|g_{n}\right|+\left|g_{n+1}\right|}{C n^{\sigma}\left|t-m_{n}(x)\right|}, 2\left(\left|g_{n}\right|+\left|g_{n+1}\right|\right)\right\} .
$$

The same holds for $t \leqq m_{n}(x)$ and for the derivatives of $f$.

(2. g. 2) For $t \in\left[b_{n+1}, b_{n}-\frac{3}{4} l_{n}\right]$, we have

$$
f=\frac{P\left(u_{n+1}+\chi_{n} u_{n}\right)}{u_{n+1}+\chi_{n} u_{n}}
$$

From (2.15), it follows that

$$
t-m_{n}(x)=t-m_{n}+m_{n}-m_{n}(x) \leqq l_{n} / 4+O\left(n^{-\sigma}\right) .
$$

Then, from (2.13), we have

$$
\begin{aligned}
\left|\frac{u_{n}}{u_{n+1}}\right| & =\exp \left(\tilde{F}_{n}\right) \\
& =\exp \left\{\left(t-m_{n}(x)\right) \partial_{t} \tilde{F}_{n}\left(m_{n}^{*}(x), x\right)\right\} \\
& \leqq \exp \left(-C n^{\sigma-\rho-1}\right) .
\end{aligned}
$$

This implies, for large $n$,

$$
\left|u_{n+1}+\chi_{n} u_{n}\right| \geqq\left|u_{n+1}\right| / 2 \text {. }
$$

Hence we conclude that

$$
\begin{aligned}
|f| & \leqq \frac{2\left(\left|g_{n+1} u_{n+1}\right|+\left|\left[P, \chi_{n}\right] u_{n}\right|+\chi_{n}\left|g_{n} u_{n}\right|\right.}{\left|u_{n+1}\right|} \\
& \leqq 2\left\{\left|g_{n}\right|+\left|g_{n+1}\right|+O\left(n^{M}\right) \exp \left(-C n^{\sigma-\rho-1}\right)\right\} .
\end{aligned}
$$

The same holds for the derivatives of $f$.

(2. g. 3) For $t \in\left[b_{n+1}+\frac{3}{4} l_{n+1}, b_{n}\right]$, we get (2.20) by the same argument as in (2. g. 2). 
From the above argument, it follows that $f$ is $C^{\infty}$ near the origin. Now we show the smoothness of $u$. From the definition of $\gamma_{n}(x)$ and (2.7), we have, for some

$$
\gamma_{n}(x) \geqq \text { const. } \cdot n^{\rho d_{1}} \text {. }
$$

On the other hand, we have, for $\mathrm{t} \in\left[b_{n+1}, b_{n-1}\right]$,

$$
\begin{aligned}
& \tau_{n}\left|t^{l+1}-b_{n}^{l+1}\right| \leqq \text { const. } \cdot n^{\rho d_{1}-1}, \\
& \left|\phi\left(\frac{t}{b_{n}}, x, b_{n}\right)\right| \leqq \text { const. } \cdot \nu_{1, n}\left|\frac{t}{b_{n}}-1\right| \leqq \text { const. } \cdot n^{\rho d_{1}-1} \cdot
\end{aligned}
$$

From these, we can easily see that $u$ is $C^{\infty}$ at $t=0$. This completes the proof of Theorem 1.2.

Remark 2.2. If $p=2$ or if the coefficients of $P$ are independent of $x$, we can take $\phi_{j}=\gamma_{j}=0$ for $j \geqq 2$. Especially in the latter case, we can take $u_{n}$ in the form:

$$
\begin{aligned}
u_{n}(t, x)= & \exp \left\{\sqrt{-1} \tau_{n}\left(\xi^{0} x-\frac{\sqrt{-1}}{l+1}\left(t^{l+1}-b_{n}^{l+1}\right)\right)\right\} \exp \left\{\phi\left(\frac{t}{b_{n}}, b_{n}\right)\right\} \\
& \times \exp \left(-\gamma_{n}\right)\left\{w_{n}\left(\frac{t}{b_{n}}\right)+z_{n}\left(\frac{t}{b_{n}}\right)\right\} .
\end{aligned}
$$

\section{References}

[1] Alinhac, S. and Zuily, C., Unicité et non unicité du problème de Cauchy pour des opérateurs hyperboliques à caractéristiques doubles, Comm. in Partial Differential Equations, 6 (1981), 799-828.

[2] Calderón, A. P., Existence and uniqueness theorems for systems of partial differential equations, Proc. Symp. Fluid Dynamics and Appl. Math. (Univ. of Maryland, 1961), Gordon and Breach, New York, (1962), 147-195.

[3] Cohen, P., The non-uniqueness of the Cauchy problem, O.N.R. Techn. Report, 93, Stanford, 1960.

[4] Hörmander, L., Non-uniqueness for the Cauchy problem, Lecture Notes in Math., Springer Verlag, 459 (1975), 36-72.

[5] Lascar, R. and Zuily, C., Unicité et non unicité du problème de Cauchy pour une classe d'opérateurs differentiels à caractéristiques doubles, Duke Math. J., 49 (1982), 137-162.

[6] Nakane, S., Uniqueness and non uniqueness in the Cauchy problem for a class of operators of degenerate type, J. Differential Equations, 51 (1984), 78-96.

[7] Nakane, S., Non-uniqueness in the Cauchy problem for partial differential operators with multiple characteristics I, Comm. in Partial Differential Equations, 9 (1984), 63-106.

[8] Nirenberg, L., Lectures on linear partial differential equations, C. B. M.S. Regional Conference series in Math., 17, A. M. S., Providence, R. I., 1972.

[9] Ōkaji, T., Uniqueness in the Cauchy problem for a class of partial differential operators 
degenerate on the initial surface, J. Math. Kyoto Univ., 24 (1984), 423-439.

[10] Plis, A., A smooth linear elliptic differential equation without any solution in a sphere, Comm. Pure Appl. Math., 14 (1961), 599-617.

[11] Roberts, G. B., Uniqueness in the Cauchy problem for characteristic operators of Fuchsian type, J. Differential Equations, 38 (1980), 374-392.

[12] Strauss, W. and Treves, F., First order linear pde's and uniqueness of the Cauchy problem, J. Differential Equations, 15 (1974), 195-209.

[13] Uryu, H., Uniqueness for the characteristic Cauchy problem and its applications, Tokyo J. Math., 5 (1982), 117-136.

[14] Zuily, C., Uniqueness and non-uniqueness in the Cauchy problem, Birkhäuser, Boston, Basel, Stuttgart, 1983. 
